\title{
A Search for Chaotic Behavior in Northern Hemisphere Stratospheric Variability
}

\author{
GuAltiero BADIN AND DANIELA I. V. DOMEISEN \\ Institute of Oceanography, University of Hamburg, Hamburg, Germany
}

(Manuscript received 23 July 2013, in final form 5 November 2013)

\begin{abstract}
Northern Hemisphere stratospheric variability is investigated with respect to chaotic behavior using time series from three different variables extracted from four different reanalysis products and two numerical model runs with different forcing. The time series show red spectra at all frequencies and the probability distribution functions show persistent deviations from a Gaussian distribution. An exception is given by the numerical model forced with perpetual winter conditions-a case that shows more variability and follows a Gaussian distribution, suggesting that the deviation from Gaussianity found in the observations is due to the transition between summer and winter variability. To search for the presence of a chaotic attractor the correlation dimension and entropy, the Lyapunov spectrum, and the associated Kaplan-Yorke dimension are estimated. A finite value of the dimensions can be computed for each variable and data product, with the correlation dimension ranging between 3.0 and 4.0 and the Kaplan-Yorke dimension between 3.3 and 5.5. The correlation entropy varies between 0.6 and 1.1. The model runs show similar values for the correlation and Lyapunov dimensions for both the seasonally forced run and the perpetual-winter run, suggesting that the structure of a possible chaotic attractor is not determined by the seasonality in the forcing, but must be given by other mechanisms.
\end{abstract}

\section{Introduction}

Climate predictability is limited by the fact that the irregular patterns observed for the variability of specific components of the climate system can be chaotic or stochastic. Chaotic systems are regulated by deterministic differential equations, nonlinearly coupled with each other, while stochastic systems are regulated by the statistical properties of the system. The study of nonlinear dynamical systems shows that it is possible to distinguish between these two forms of variability from the analysis of the time series of a single variable (Grassberger and Procaccia 1983a,c). Deterministic dynamical systems are associated with the presence of an attractor set characterized by a finite, even though fractal, dimension and a converging value of the entropy of the system, which represents a measure of the uncertainty associated with the measurement of a state. Stochastic systems are instead characterized by a lack of convergence to a finite dimension and dynamical entropy. In addition, chaotic systems are characterized by

Corresponding author address: Gualtiero Badin, Institute of Oceanography, University of Hamburg, Bundesstrasse 53, D-20146 Hamburg, Germany.

E-mail: gualtiero.badin@zmaw.de sensitivity to initial conditions and, thus, by the presence of exponentially divergent trajectories on the strange attractor. The coefficient of the exponential divergence of trajectories that were initially infinitesimally close takes the name of Lyapunov exponents. The time series analysis of finite-size Lyapunov exponents yields information on the predictability of slow-varying dynamics, corresponding to large-scale flows in geophysical systems, when the fast-varying dynamics are unresolved (Aurell et al. 1997; Boffetta et al. 1998).

While these mathematical features are certainly attractive for the characterization of the variability of the climate system, their application to uncontrolled experiments has a problematic history. Using observations, the existence of an attractor for climate was instead studied from different sources of data, ranging from ${ }^{18} \mathrm{O} /{ }^{16} \mathrm{O}$ ratio in deep sea cores (Nicolis and Nicolis 1984; Vautard and Ghil 1989) to local surface variables such as local surface pressure, relative sunshine duration, and zonal wave amplitude (e.g., Fraedrich 1986, 1987; Zeng et al. 1992; Weber et al. 1995). While some of these studies found a strange attractor with a finite dimension, ranging from 3 to 13 , others did not find signs of deterministic chaos (the same problem appears for other natural systems: see, e.g., the study by Carbonell et al. 1994 for the search for deterministic chaos in solar 
activity). In particular, the positive results were criticized by Grassberger (1986) and Ruelle (1990) [in turn criticized in an analytical study by Essex and Nerenberg (1991)], who addressed the problem of sparse data as well as the errors of the estimates. An argument against the characterization of deterministic versus stochastic variability through the determination of a finite correlation dimension was suggested by Osborne and Provenzale (1989) [subsequently criticized in an analytical study by Theiler (1991)], who showed that a finite dimension can be found in systems that are not deterministic but that possess random, self-similar time series that exhibit fractional Brownian motion (Mandelbrot and VanNess 1968). A further problem was highlighted by Lorenz (1991), who showed that, using a system of 21 ordinary differential equations, the dimension of the attractor determined from the analysis of the time series of one of the variables is lowered by sparse data and depends on the chosen variable. Even if these results seemed to preclude the determination of the existence of an attractor for the climate system, Lorenz (1991), followed by Elsner and Tsonis (1992) and Tsonis et al. (1993), proposed that the atmosphere might be viewed as a loosely coupled system and that the finite attractor dimensions found by some of these studies might indeed correspond to the dimension of a subsystem. In this study we again raise the question of the existence of a finite attractor, but guided by the studies described above we restrict our attention to a climate subsystem, represented by the Northern Hemisphere extratropical stratosphere.

The dominant time scale in the extratropical stratosphere is the seasonal cycle, since the dynamical evolution of the stratosphere is strongly dependent on available sunlight. The seasonal lack of sunlight leads to a strong meridional temperature gradient, which is particularly strong at the edge of the polar night. There, the polar vortex forms in autumn and reaches its maximum strength in midwinter, but weakens to a complete wind reversal in late spring. While radiative processes maintain the polar vortex, planetary-scale Rossby waves (dominantly zonal wavenumbers $1-3$ ), which are dominantly caused by longitudinally asymmetric heating and topography at Earth's surface, cause strong disruptions of the vortex in so-called stratospheric sudden warming (SSW) events [first described in Scherhag $(1952,1953)$, and documented in, e.g., Labitzke (1981)]. These events are observed in midwinter as abrupt changes in the stratospheric wind, temperature, and geopotential height patterns. The presence of large-scale waves thus introduces variability on shorter time scales to the winter stratosphere: the time scale on which waves are able to propagate and interact with the mean flow (on the order of days) and the radiative time scales on which the flow recovers to radiative equilibrium (on the order of weeks). The large wave amplitudes throughout Northern Hemisphere winter are able to consistently weaken the polar vortex, thereby keeping the vortex from reaching wind speeds comparable to its Southern Hemisphere counterpart, where vortex wind speeds grow continuously into winter and wave amplitudes exhibit a minimum in midwinter (Hirota et al. 1983), indicating that wind speeds may be too strong even for zonal wave 1 to propagate (Plumb 1989). The spring transition in both hemispheres is dominated by the final warming, which is induced by both radiative forcing and tropospheric waves (see, e.g., Black et al. 2006). The summer circulation, on the other hand, is comparably quiet, as Rossby waves are not able to propagate through the easterly winds which dominate the summer stratosphere (Charney and Drazin 1961). This behavior leads to a significant difference in variability between the summer and winter season in the stratosphere.

The chaotic behavior of the stratospheric variability has been investigated using the low-dimensional HoltonMass model (Holton and Mass 1976) forced by a timeindependent forcing at the lower boundary by Yoden (1987a,b, 1990) and Christiansen (2000). Analyzing the bifurcations of the Holton-Mass model, Yoden (1987a,b, 1990) showed the existence of a quasi-periodic state branching off the periodic vacillations through the presence of a Hopf bifurcation. Further analysis (Christiansen 2000) showed a route to chaos determined by the consecutive presence of three Hopf bifurcations. A simple prototype of SSW events was also studied in a highly truncated version of the Holton-Mass model by Ruzmaikin et al. (2003). Considering that external forcing, such as baroclinic instabilities in the troposphere or gravity waves, can act as a stochastic forcing to the stratospheric variability, the low-order model by Ruzmaikin et al. (2003) was modified to include stochastic forcing by Birner and Williams (2008). Their study found that even small to moderate strengths of the stochastic gravity wave forcing can be sufficient to cause a SSW event for cases for which the deterministic system would not have predicted a SSW event. The Holton-Mass model was modified by Hardiman and Haynes (2008) to include the effect of upper level forcing. The vortex response associated with SSW events was studied as a nonlinear forced oscillator by Esler and Matthewman (2011). For a review of these studies on stratospheric variability, see Haynes (2005).

This study investigates the variability of the extratropical stratosphere at $10 \mathrm{hPa}$ and $60^{\circ} \mathrm{N}$, corresponding to the location where the WMO threshold for SSW events 
is defined (McInturff 1978). Zonal wind, temperature, and geopotential height data are analyzed in four reanalysis datasets. To further evaluate the difference between winter and summer variability, idealized model simulations were performed: one forced with a seasonal cycle (to evaluate the difference between the idealized model and the reanalysis data), and one model simulation forced with perpetual winter conditions (to assess the role of the seasonal cycle in determining the structure of the chaotic attractor).

Given the availability of long time series for different variables allows us to address the following questions: 1) Is there a finite attractor for a specific climate subsystem, here represented by stratospheric variability? 2) Do the dimension and characteristics of a possible attractor depend on the choice of the analyzed variable, as found by Lorenz (1991) through the study of a mathematical attractor?

\section{Theoretical background}

\section{a. Phase space embedding}

Given a set of partial differential equations, if the system can be expanded into $n$ orthogonal functions, it is possible to transform it into a set of $n$ ordinary differential equations in the expansion coefficients $x_{i}$ (e.g., Lorenz 1960):

$$
\dot{x}_{i}=f_{i}\left(x_{1}, \ldots, x_{n}\right), \quad i=1, \ldots, n,
$$

where the dot on the $x_{i}$ indicates a time derivative. The resulting trajectories span an $n$-dimensional phase space forming an invariant manifold. If the system is chaotic, the invariant manifold is called a strange attractora geometric object characterized by a fractal dimension $d<n$ that can be a noninteger (e.g., Mandelbrot 1977). If all but one variable $x(t)$ are eliminated from (1), the system is reduced to a nonlinear differential equation of order $n$ :

$$
x^{(n)}=f\left[x, x^{\prime}, \ldots, x^{(n-1)}\right],
$$

where primes indicate differentiation. The system spans a phase space that can be embedded in an $m=2 d+1$ dimensional space created by the discretization of the nonlinear differential equation and defined by the delay coordinates

$$
\mathbf{x}(t)=\{x(t), x(t+\tau), \ldots, x[t+(m-1) \tau]\},
$$

where $\tau$ is a time lag (Whitney 1936; Takens 1981). The choice of a variable $x(t)$ has thus the potential to reduce the problem to the analysis of a time series of only one variable of the given system (i.e., the time series of an observable).

\section{b. Dimensions and entropies}

The dimension of a strange attractor corresponds to the Hausdorff dimension, which has an upper bound in the box-counting dimension. Considering a set in $\mathbf{R}^{m}$, if the space is covered with a regular grid of boxes of length $\epsilon$ and if $N(\epsilon)$ is the number of boxes that contain at least one point of the set, self-similarity implies $N(\epsilon) \propto \epsilon^{-D_{0}}$, where $D_{0}$ is the box-counting dimension. Attractors can be described in more detail by their measures - that is, the probability distribution to find a trajectory in a subset of the attractor, or, more heuristically, a quantity describing how frequently different parts of the attractor are visited by the trajectories of the dynamical system considered (Eckmann and Ruelle 1985). For a strange attractor with a nonhomogeneous measure it is possible to give a formal generalization of the concept of dimension in order to give more weight to the parts of space that are visited more frequently by the dynamics. Following Renyi (1971), given a dynamical system with measure $\mu$, and denoting with $p_{\epsilon}(\mathbf{x})$ the probability to find a trajectory in a ball $\mathcal{U}_{\epsilon}(\mathbf{x})$ of radius $\epsilon$ around $\mathbf{x}$, it is possible to define the correlation integral

$$
C_{q}(\epsilon)=\int_{\mathbf{x}} p_{\epsilon}^{q-1} d \mu(\mathbf{x}) \equiv\left\langle p_{\epsilon}^{q-1}\right\rangle_{\mu} .
$$

If the attractor is self-similar, (4) satisfies

$$
C_{q}(\epsilon) \propto \epsilon^{(q-1) D_{q}}, \quad \epsilon \rightarrow 0 .
$$

Equation (5) defines a family of $q$ dimensions (Hentschel and Procaccia 1983)

$$
D_{q}=\lim _{\epsilon \rightarrow 0} \frac{1}{q-1} \frac{\ln C_{q}(\epsilon)}{\ln \epsilon} .
$$

The solution with $q=0$ yields the capacity dimension, obtained by covering the attractor with a regular grid of $N$ boxes of length $\epsilon$, so that $\left\langle p_{\epsilon}\right\rangle_{\mu}=1 / N(\epsilon)$ and $D_{0}=$ $-\lim \ln N(\epsilon) / \ln \epsilon ; q=1$ yields the information (or Shannon) dimension $D_{1}=\lim _{\epsilon \rightarrow 0}\left\langle\ln p_{\epsilon}\right\rangle_{\mu} / \ln \epsilon$. Finally, for $q>1$, (4) can be estimated $\stackrel{\epsilon \rightarrow 0}{\rightarrow} 0$ the correlation $C_{q}(m)$ between the points obtained by the projection of the delay vectors (3) in the $m$-dimensional embedding space. The correlation integral can be calculated as

$$
C_{q}(m, \epsilon)=\frac{1}{N(N-1)^{(q-1)}} \sum_{i=1}^{N}\left[\sum_{i \neq j} \Theta\left(\epsilon-\left\|\mathbf{x}_{i}-\mathbf{x}_{j} \mid\right\|\right)\right]^{q-1},
$$


where $\Theta(x)$ is the Heaviside function so that $\Theta(x)=1$ if $x>0$ and $\Theta(x)=0$ if $x \leq 0$. Equation (7) can be calculated for the case $q=2$ making use of an Euclidean norm (Grassberger and Procaccia 1983a,c), for which case $D_{2}$ assumes the name of correlation dimension:

$C_{2}(m, \epsilon)=\frac{1}{N(N-1)} \sum_{i=1}^{N}\left[\sum_{i \neq j} \Theta\left(\epsilon-\left\|\mathbf{x}_{i}-\mathbf{x}_{j}\right\|\right)\right]$.

After computation of $C_{2}$ as a function of $\epsilon$ for $m=1, \ldots$, $M$, the correlation dimension can be calculated from (6) as the slope

$$
D_{2}=-\frac{\partial \ln C_{2}(\epsilon)}{\partial \ln \epsilon} .
$$

A convergence of $D_{2}$ for increasing values of $m$ at a dimension $D_{2} \ll M$ indicates the presence of a finite correlation dimension. It is possible to prove that $D_{2}$ represents a lower bound for $D_{1}$, where $D_{2}$ is easier to calculate for a limited amount of data (Grassberger and Procaccia 1983a).

If now the phase space is partitioned in disjoint boxes $\mathcal{P}_{j}$ and $p_{j}=\int_{\mathcal{P}_{j}} d \mu(\mathbf{x})$ is the fraction of the measure contained in the jth disjoint box, it is possible to define the order- $q$ Renyi entropies (Grassberger and Procaccia 1983b)

$$
h_{q}=-\lim _{\tau \rightarrow 0} \lim _{\epsilon \rightarrow 0} \lim _{m \rightarrow \infty} \frac{1}{\tau m(q-1)} \ln \sum_{i_{1}, \ldots, i_{m}} p^{q}\left(i_{1}, \ldots, i_{m}\right),
$$

which can be interpreted as the time rate of loss of information as the trajectories evolve (Eckmann and Ruelle 1985). Using $q=1$ in (10) defines the KolmogorovSinai entropy (Kolmogorov 1958; Sinai 1959) that is null for ordered systems, finite for deterministic chaotic systems, and infinite for stochastic systems. Using (7), and with $q=2$ one obtains

$$
h_{2}(m, \epsilon)=-\ln \frac{C_{2}(m, \epsilon)}{C_{2}(m+1, \epsilon)},
$$

which can be interpreted as the number of pairs of trajectories that escape $\mathcal{U}_{\epsilon}(\mathbf{x})$ when $m$ is increased to $m+1$. While the determination of $h_{1}$ is often limited by sparse data, it is possible to prove that $h_{1} \geq h_{2}$ (Grassberger and Procaccia 1983b). The calculation of $h_{2}$ allows thus for the determination of a lower bound for the entropy of the system.

\section{c. Lyapunov exponents and relation between dimensions}

The Lyapunov exponents are the coefficients of exponential separation of neighboring trajectories on a strange attractor and are thus representative of the sensitivity to the initial conditions of the system. The spectra of Lyapunov exponents are here calculated following the algorithm of Sano and Sawada (1985). The number of Lyapunov exponents is the same as the dimension of the embedding phase space, while the ordered Lyapunov spectra $\lambda_{1} \geq \cdots \geq \lambda_{m}$ define the dimension (Kaplan and Yorke 1979)

$$
D_{\mathrm{KY}}=j+\frac{1}{\left|\lambda_{j+1}\right|} \sum_{i=1}^{j} \lambda_{i},
$$

where the sum is over the first $j$ nonzero Lyapunov exponents. Equation (12) is referred to as Kaplan-Yorke, or Lyapunov, dimension. It is possible to prove (Grassberger and Procaccia 1983c) that the $D_{q}$ are nonincreasing functions of $q$, so that the different dimensions listed are linked by the relationship

$$
D_{\lambda} \leq D_{2} \leq D_{1} \leq D_{0} \leq D_{\mathrm{KY}},
$$

where $D_{\lambda}$ is the number of positive Lyapunov exponents. Systems in which the equalities in (13) hold are called monofractals, while systems that possess large differences between the different dimensions are called multifractals. The hierarchy of dimensions and entropies allows for the calculation of lower and upper bounds for the dimension and entropy of the chaotic attractor, when the direct calculation of the box-counting and information dimension, as well as the Kolmogorov-Sinai entropy, are strongly affected by the limitations in the length of the time series.

\section{d. Practical considerations regarding the calculation of the dimension of the attractor}

The determination of the box-counting dimension $D_{0}$ is particularly difficult to determine owing to the need to create boxes of infinitesimal size in order to cover the strange attractor. At the same time, the information dimension $D_{1}$ is also affected by finite-size datasets. The correlation dimension $D_{2}$ will thus be calculated. Each time series will be analyzed separately. To embed the time series, different choices for the embedding dimension and the time delay can be made. For a summary of the possible choices see, for example, Tsonis et al. (1993). In this study, the maximum embedding dimension will be defined as (Ruelle 1990)

$$
M=2 \log _{10} N,
$$

where $N$ is the number of data points in each time series. It can be noted that the logarithmic growth of the required embedding dimension represents a major obstacle 
in the embedding of the system. In fact, the arguments produced by Grassberger (1986) and Ruelle (1990) against some of the results found for the climate attractor rely on the observation that some of the studies that claimed to find a finite attractor for climate used a maximum embedding dimension larger than the value predicted by (14).

The delay time will instead be evaluated as the first minimum of the mutual information (Fraser and Swinney 1986). Once each time series is embedded, the correlation integrals are calculated varying the values of the embedding dimension from $m=1$ to $m=M$ and calculating the slopes (9). One hundred values of $\epsilon$ are used between $N_{\epsilon} / 1000$ and $N_{\epsilon}$, where $N_{\epsilon}$ is the maximum interval between the data. The slopes are calculated using central differences. For deterministic chaos, the slopes must converge to the same value of $D_{2}$ for a large enough interval of $\epsilon$.

\section{Data}

The variability in time of the extratropical stratosphere is analyzed using daily mean values of three different variables extracted from four reanalysis datasets and two different idealized model simulations. The three variables examined are the daily and zonal mean zonal wind, temperature, and geopotential height. All quantities are evaluated at $10 \mathrm{hPa}$ and at the grid point closest to $60^{\circ} \mathrm{N}$.

\section{a. Reanalysis data}

The following reanalysis datasets are used for the analysis: the Interim European Centre for MediumRange Weather Forecasts (ECMWF) Re-Analysis (ERA-Interim) (Dee et al. 2011) from 1 January 1980 to 31 December 2011, yielding a total of 11688 daily data points; the 40-yr ECMWF Re-Analysis (ERA-40) reanalysis (Uppala et al. 2005) from 1 January 1958 to 31 December 2001, yielding a total of 16071 data points; the National Centers for Environmental Prediction (NCEP) reanalysis (Kalnay et al. 1996) from 1 January 1948 to 31 December 2012, yielding a total of 23742 points; and the NCEP-U.S. Department of Energy (DOE) Atmospheric Model Intercomparison Project II (AMIP-II) reanalysis (R-2) dataset (Kanamitsu et al. 2002) from 1 January 1979 to 31 December 2012, yielding a total of 12419 data points. The seasonal cycle has been removed from each reanalysis time series by subtracting the climatological seasonal cycle for leap years and nonleap years separately.

\section{b. Model data}

The model used for this study is the Geophysical Fluid Dynamics Laboratory (GFDL) three-dimensional atmospheric spectral dynamical core model at T42 resolution on 40 hybrid $\sigma$-pressure levels up to $0.02 \mathrm{hPa}$ (with 28 levels above $200 \mathrm{hPa}$ ) and a sponge layer starting above $0.8 \mathrm{hPa}$. The model uses a Newtonian relaxation to a zonal mean equilibrium temperature profile based on Held and Suarez (1994) in the Polvani and Kushner (2002) setup (using $\gamma=4 \mathrm{~K} \mathrm{~km}^{-1}$ and $\epsilon=-10 \mathrm{~K}$ ). The relaxation time scale is 40 days in the stratosphere. Zonal wave-2 topography of height $3000 \mathrm{~m}$ is used to force stratospheric variability as defined in Gerber and Polvani (2009).

Two model runs are performed: model I is forced with a 360-day seasonal cycle (as defined in Kushner and Polvani 2006), while model II is run without a seasonal cycle (i.e., in perpetual winter conditions for the analyzed hemisphere). The specific run performed for model I is documented in Sheshadri et al. (2014), while the run performed for model II is described in Gerber and Polvani (2009) (their run 9), except here run on hybrid levels (instead of $\sigma$ levels). The main limitation of this model setup is the structure of the equilibrium temperature profile in the tropical lower stratosphere, in particular the location of the tropical tropopause, and an improved equilibrium temperature profile has been designed by Jucker et al. (2013) to address this issue. However, the stratospheric variability at the stratospheric location chosen for this particular study is only affected to a minor extent by the improved equilibrium temperature profile.

Model I is run for 13000 days, and the last 33 yearly cycles are used for the analysis. The seasonal cycle is removed by subtracting the 360-day climatological seasonal cycle from each model year. Model II was run for 29900 days. Results are reported as daily mean values for both model runs.

\section{Results}

\section{a. Variability in the different datasets}

\section{1) TIME SERIES VARIABILITY AND TRENDS}

By removing the seasonal cycle from the presented time series, the characteristic change in stratospheric variability between summer and winter season becomes more obvious. Figure 1 shows the time series of zonal mean zonal wind at $60^{\circ} \mathrm{N}$ and $10 \mathrm{hPa}$ for all datasets. Sudden (in midwinter) and final (at the end of winter) stratospheric warming events can be observed as sudden drops in the wind. The NCEP data shows a positive temperature trend of $\approx 8^{\circ} \mathrm{C}$ in the first 50 years, which is reflected in a positive trend in geopotential height, followed by a negative trend of $\approx 2.5^{\circ} \mathrm{C}$ in the remainder of the time series (not shown). The early positive trend in 
(a)
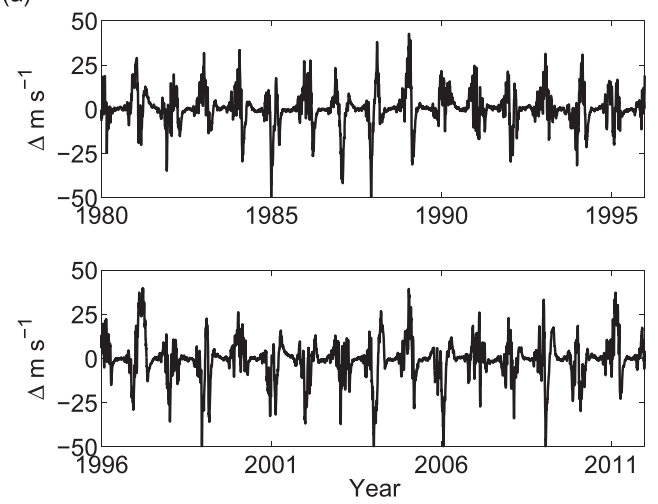

(c)
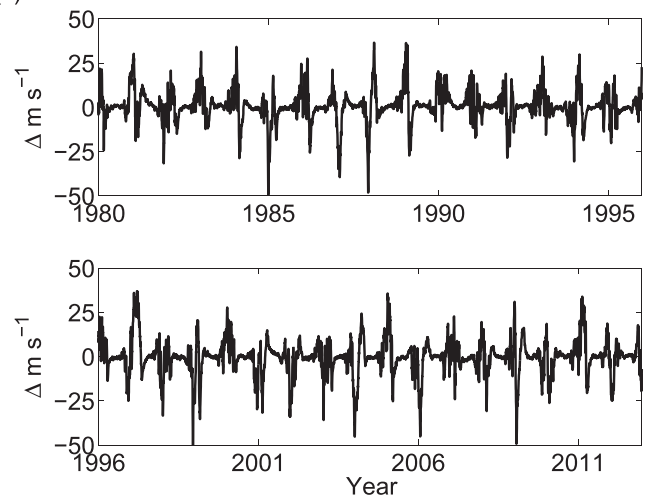

(e)
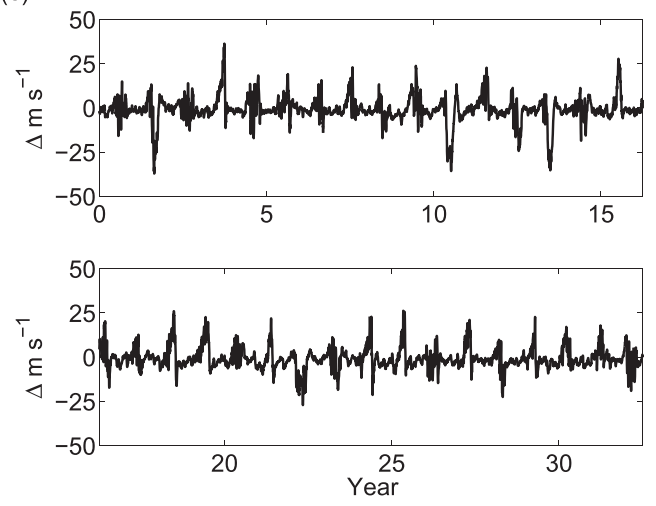

(b)
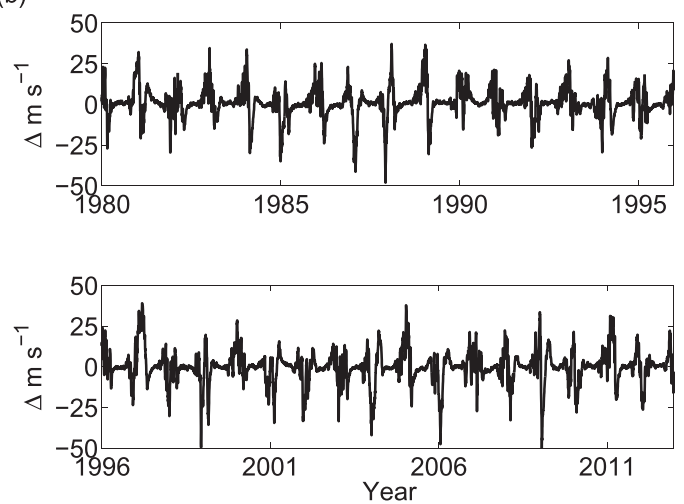

(d)
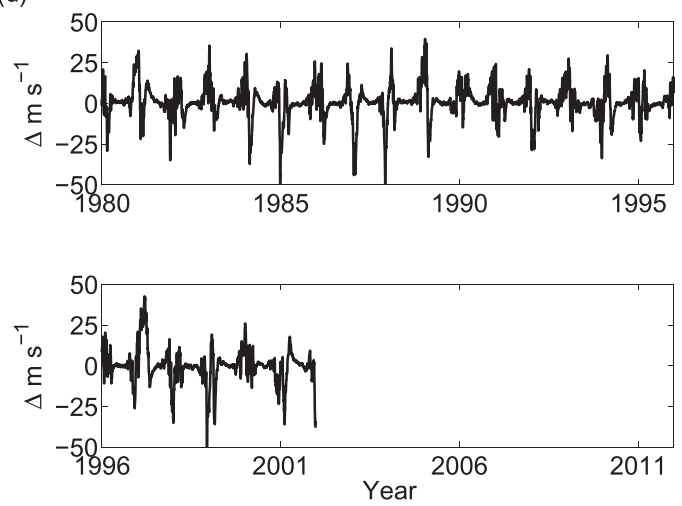

(f)
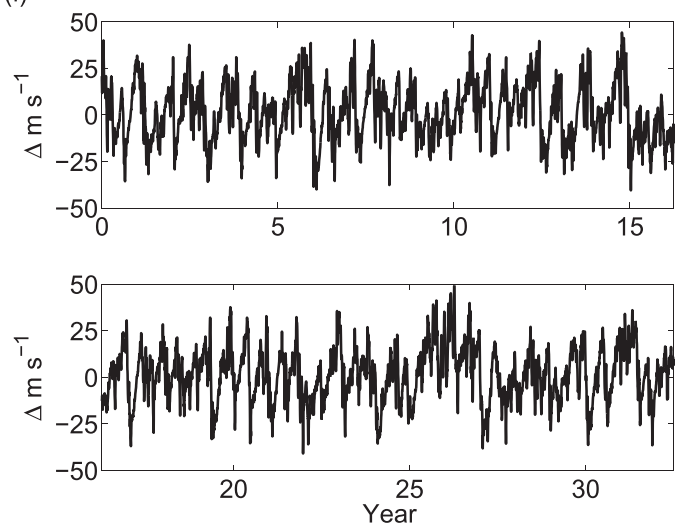

FIG. 1. Time series of zonal mean zonal wind at $60^{\circ} \mathrm{N}$ and $10 \mathrm{hPa}$ between 1980 and 2011 for (a) ERA-Interim, (b) NCEP, (c) NCEP2, and (d) ERA-40; and (e) model I with the seasonal cycle removed, and (f) model II with the time mean subtracted. The NCEP, NCEP2, and ERA-40 time series have been cropped in order to show the same interval of years as ERA-Interim. Model II has been cropped in order to allow for better comparison with model I. As expected, stratospheric variability is considerably larger during winter as compared to the summer season. Model II shows a continuous strong variability as expected during permanent winter conditions.

the NCEP data is likely due to inconsistencies in the early measurements, as documented in Kistler et al. (2001), while the negative trend is likely due to sensitivity to climate change. A positive drift can also be observed in the ERA40 data for the early years of the reanalysis (not shown).
Model II shows a frequency of SSWs corresponding to one major warming about every 200 days, which compares very well with the frequency in reanalysis data of six SSWs per decade, considering the level of complexity of the model. Because model II is forced by permanent 
(a)

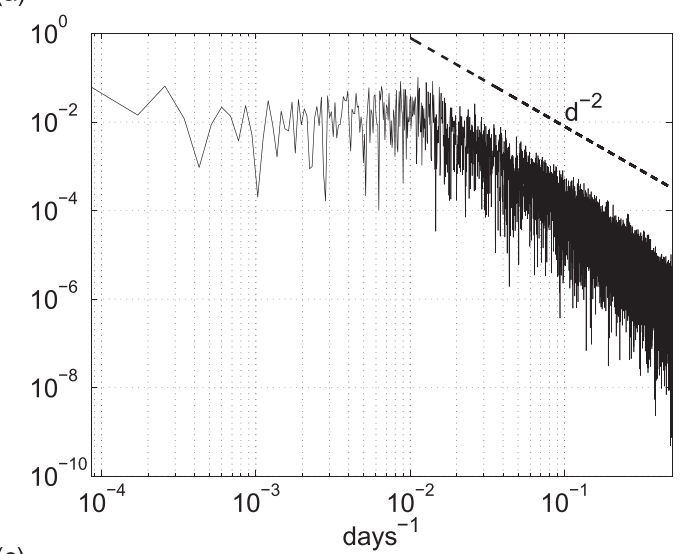

(c)

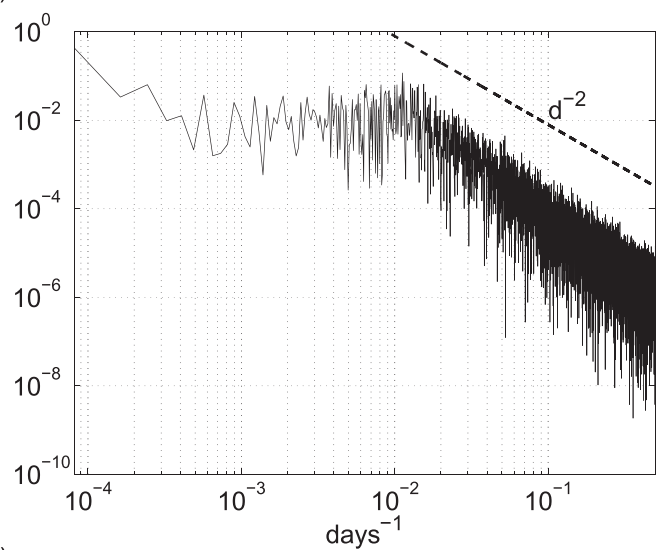

(e)

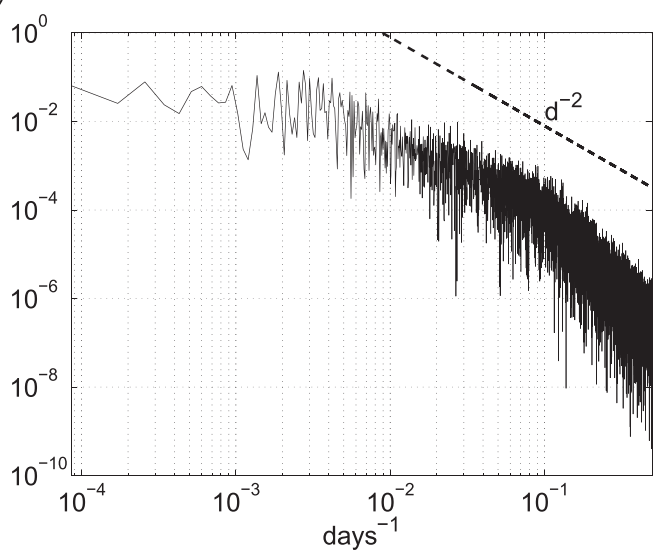

(b)

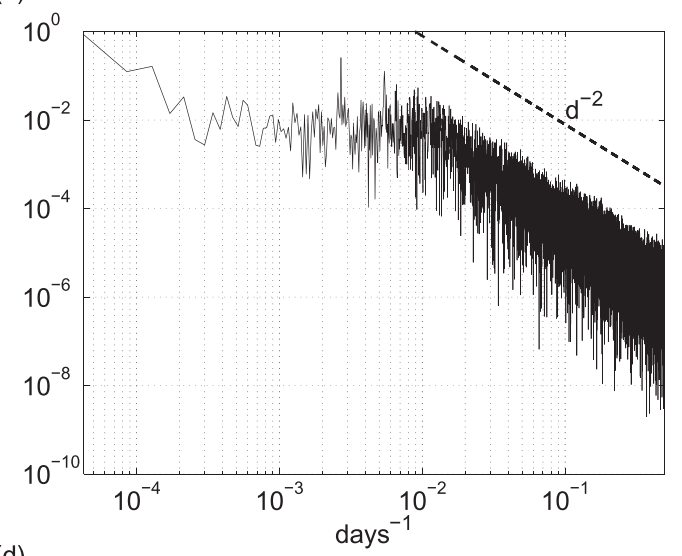

(d)

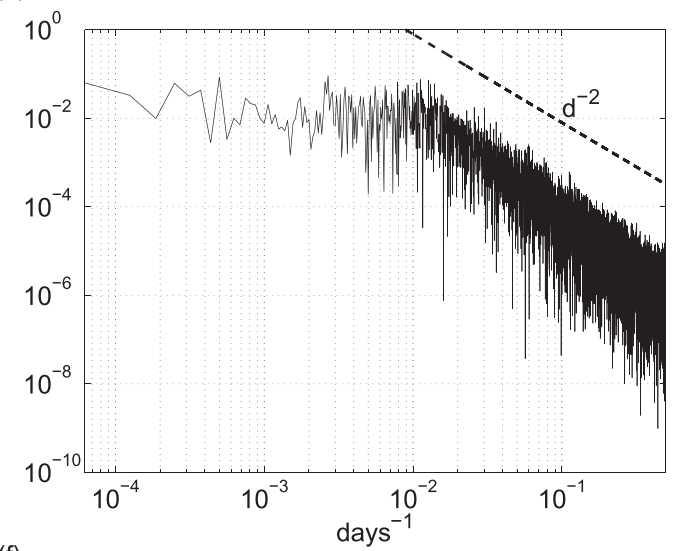

(f)

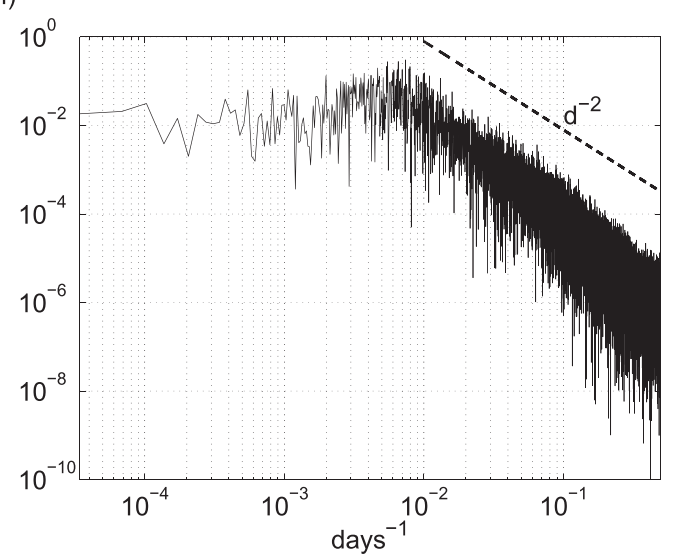

FIG. 2. Frequency spectra for zonal mean temperature at $60^{\circ} \mathrm{N}$ and $10 \mathrm{hPa}$ for (a) ERA-Interim, (b) NCEP, (c) NCEP2, (d) ERA-40, (e) model I, and (f) model II. Dashed lines with slope -2 are plotted for comparison.

winter conditions, it shows continuous variability throughout the time series (Fig. 1).

\section{2) FREQUENCY SPECTRA}

The frequency spectra of the respective time series are more difficult to distinguish between the different datasets. The zonal mean temperature spectra for the reanalyses and the two model simulations are shown in
Fig. 2. Generally, a red spectrum with slope -2 (dashed lines) can be observed for all the time series beyond a cutoff frequency of about $10^{-2} \mathrm{cpd}$, which is in contrast to the troposphere where a white spectrum dominates beyond a cutoff frequency of about $0.4 \mathrm{cpd}$ (Keppenne and Nicolis 1989). The cutoff frequency of about $10^{-2} \mathrm{cpd}$ is in agreement with the value found in the idealized modeling studies by Scott and Polvani 
(a)

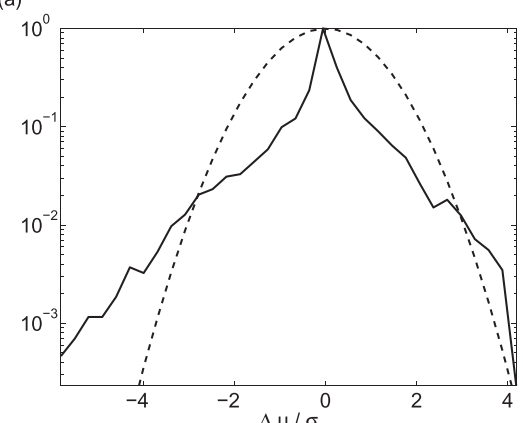

(d)

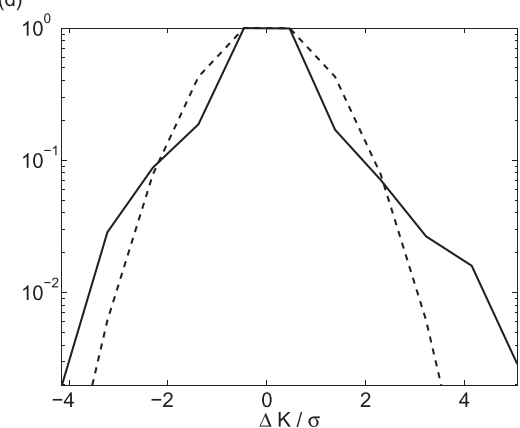

(g)

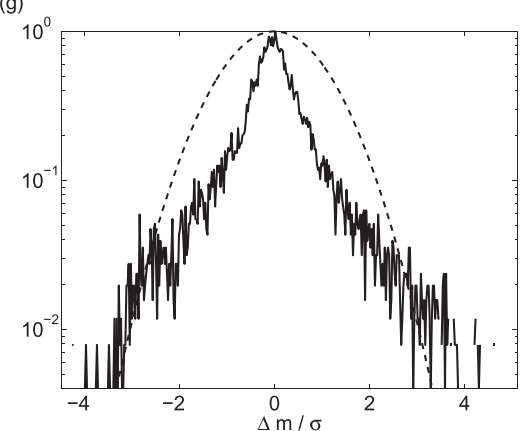

(b)

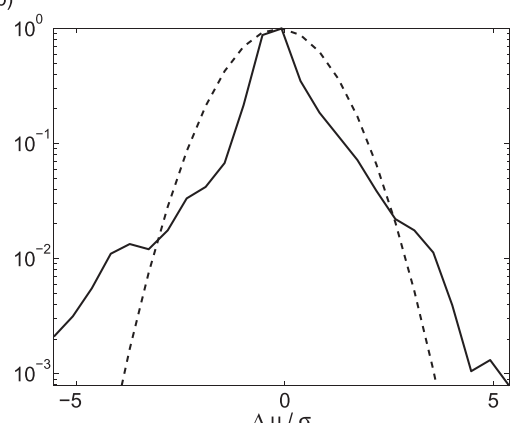

(e)

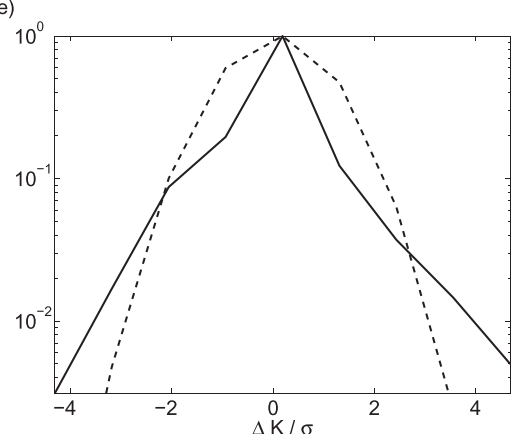

(h)

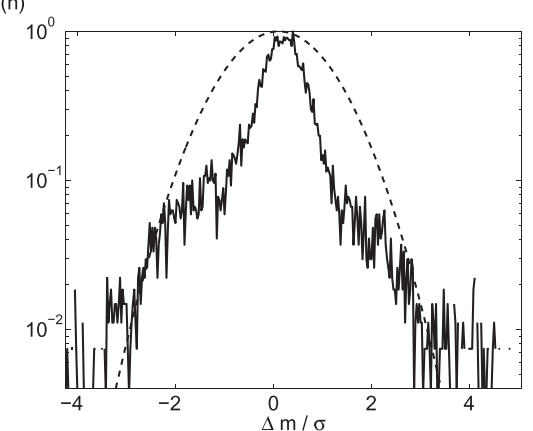

(c)

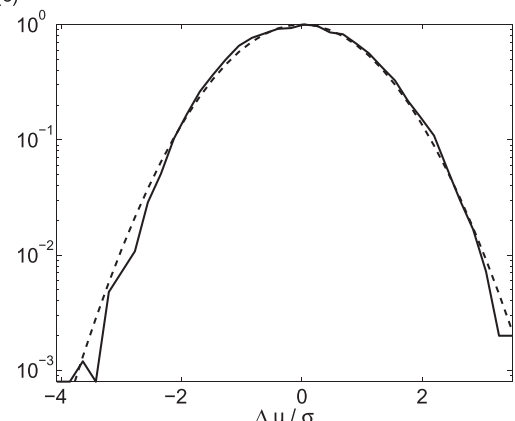

(f)

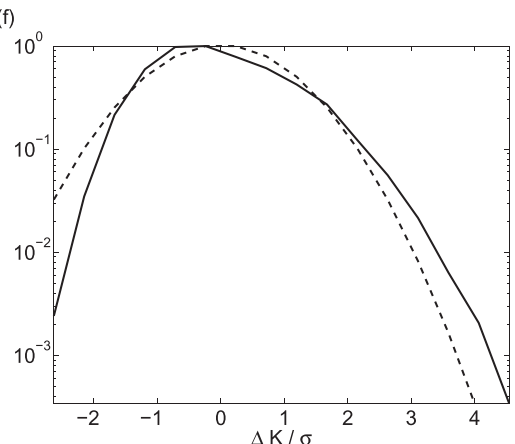

(i)

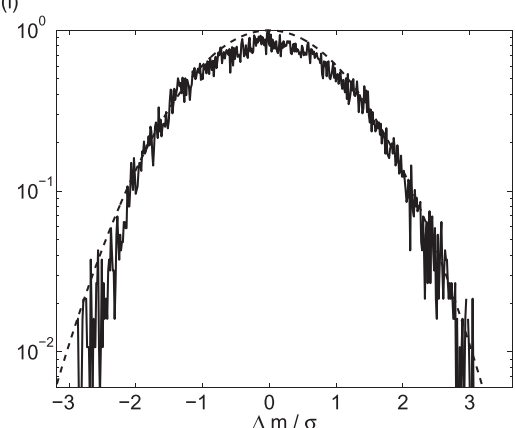

FIG. 3. PDFs of the zonal mean (a)-(c) zonal wind, (d)-(f) temperature, and (g)-(i) geopotential height for (left) ERA-Interim, (center) model I, and (right) model II. The dashed line indicates a normal distribution. While the reanalysis and model I show deviations from the Gaussian profiles for all variables, the PDFs of zonal mean zonal wind and zonal mean geopotential height for model II closely follow a Gaussian distribution.

(2006) and Scott et al. (2008) that find dominant time scales for the stratospheric frequency spectrum of about 100 days even when forcing the system with shorter time scales, thus suggesting that the stratosphere, at least to a certain extent, determines its own internal variability.

Despite the removal of the annual cycle, the NCEP temperature and geopotential height spectra (not shown) show a peak around the yearly cycle. The peak disappears in the spectra for the subinterval from 1 January 1979 to 31 December 2012, which eliminates the positive temperature trend over the first $50 \mathrm{yr}$.

Model I shows a more complex spectral structure, with a flatter slope between -1 and -2 between $10^{-3}$ and $10^{-1} \mathrm{cpd}$ and a slope of less than -2 for higher frequencies. Model II reflects the higher variability produced by permanent winter forcing.

\section{3) PRobABILITY DENSITY FUNCTIONS}

The structure of the variability can be studied through the probability density functions (PDFs) of the different variables (Fig. 3). In all the reanalyses and model I, the PDF profiles for all variables show persistent deviations from the Gaussian distribution. The deviations show a large number of events within the Gaussian tails with an asymmetry between positive and negative values in the PDF. The different variables do not differ significantly from each other in the distribution except for the noisier distribution for geopotential height. The PDFs of the mean zonal mean wind and of the zonal mean 
geopotential height for model II are instead close to the corresponding normal distributions. Deviation from Gaussianity has been shown to hold in the atmosphere at different spatial and time scales (e.g., Christiansen 2009). The deviations from Gaussianity could be either related to the asymmetry between positive and negative deviations from the climatological mean (i.e., stratospheric weak and strong vortex events) as documented in, for example, Baldwin and Dunkerton (2001), or to the difference between the summer and winter circulation, where the summer circulation yields values closer to the mean of the yearly average, while in winter variability is considerably higher and therefore yields larger deviations from the mean. To determine which of the two hypotheses for the deviation from Gaussianity holds true, the PDFs for the ERA-Interim zonal mean zonal wind are studied separately for winter, defined as December-February (DJF; Fig. 4a), and summer, defined as June-August (JJA; Fig. 4b). Both PDFs closely follow the Gaussian distribution, suggesting that the deviations from Gaussianity are due to the transition between summer and winter variability. This confirms the result from model 2, which is forced with permanent winter conditions and where the lack of transition from winter to summer variability results in PDFs that follow distributions that are very close to Gaussianity. The asymmetry between strong and weak vortex events can instead be the reason for the slight asymmetry of the winter PDF (Fig. 4a) toward a stronger tail for weak winds.

Even if deviations from Gaussianity are not enough to draw conclusions about the presence of deterministic chaos, some chaotic time series show different higherorder statistics from Gaussian signals (e.g., Vautard and Ghil 1989). To determine the existence of an attractor of stratospheric variability, we will now determine the existence of finite correlation and Lyapunov dimensions.

\section{b. Determination of the dimensions and entropy of the attractor}

\section{1) MAXIMUM EMBEDDING DIMENSIONS AND TIME DELAYS OF THE TIME SERIES}

For the different datasets considered in this study, the maximum embedding dimension $M$ varies between 8.13 and 8.95. The value of $M=8$ will thus be used throughout the study. The delay time will instead be evaluated as the first minimum of the mutual information (Fraser and Swinney 1986). Because model II does not show a minimum in the mutual information, for this dataset the first zero of the autocorrelation function has been used. Because good practice requires a variation of the delay time to check for the consistency of the results, the first zero of the autocorrelation function has been used to (a)

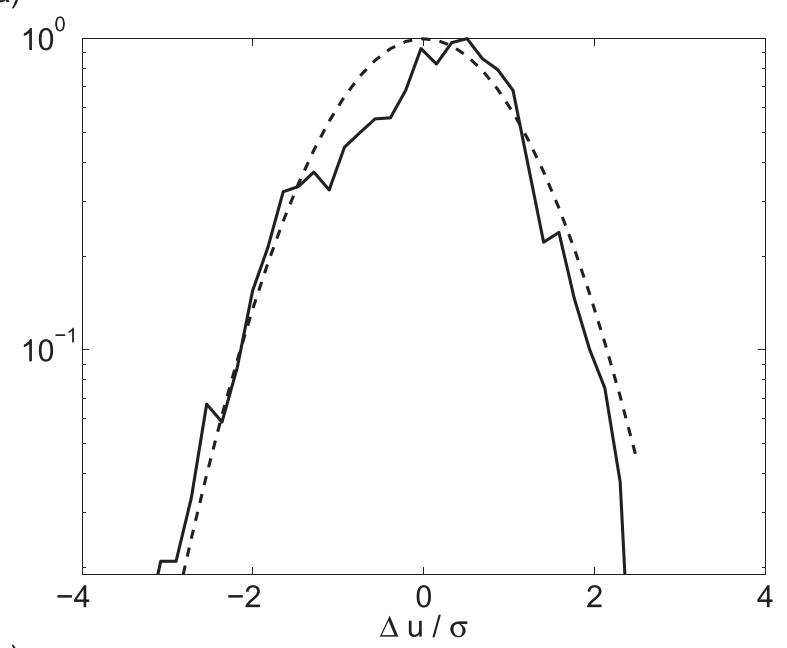

(b)

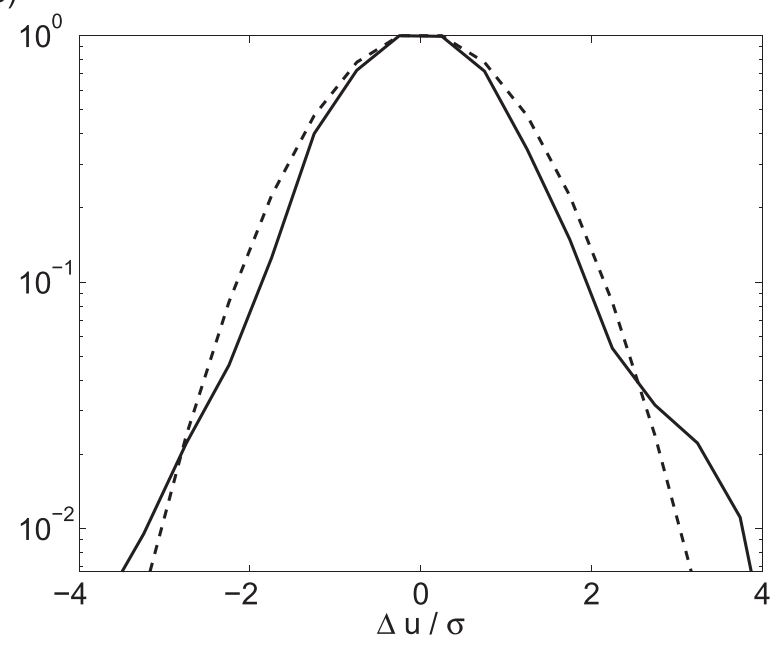

FIG. 4. PDFs of the zonal mean zonal wind for ERA-Interim for (a) JJA and (b) DJF. The dashed line indicates a normal distribution. The distributions closely follow the Gaussian distribution as suggested by the PDFs of model II, indicating that the deviations from Gaussianity are due to the change in variability between winter and summer.

compare the results with those obtained using the first minimum of the mutual information. The values of $\tau$ used for the different variables and datasets are reported in Table 1, column 1. Results show that the first minimum of the mutual information varies between 61 and 109 days. The values obtained from the first zero of the autocorrelation function for model II are instead much lower and vary between 38 and 64 days. Notice that these values are different from the values that are usually given for the decorrelation time in the stratosphere, which are determined as the $e$-folding time scale-that is, the time when the autocorrelation function decreases to 1/e [as discussed in, e.g., Baldwin et al. (2003) and Gerber et al. (2008)]. 
TABLE 1. Number of positive Lyapunov exponents $\left(D_{\lambda}\right)$, correlation dimension $\left(D_{2}\right)$, Kaplan-Yorke dimension $\left(D_{\mathrm{KY}}\right)$, maximum Lyapunov exponent $\left(\lambda_{\max }\right)$, and correlation entropy $\left(h_{2}\right)$ for zonal mean zonal wind, temperature, and geopotential height for the reanalyses and model time series.

\begin{tabular}{|c|c|c|c|c|c|c|}
\hline & $\tau$ & $D_{\lambda}$ & $\mathrm{D}_{2}$ & $D_{\mathrm{KY}}$ & $\lambda_{\max }$ & $h_{2}$ \\
\hline \multicolumn{7}{|l|}{ Zonal mean zonal wind } \\
\hline ERA-Interim (mutual information) & 97 & 3 & $3.5 \pm 0.2$ & 5.3 & 0.084 & $0.9 \pm 0.3$ \\
\hline ERA-Interim (autocorrelation) & 40 & 3 & $3.2 \pm 0.4$ & 5.3 & 0.082 & $>0.9$ \\
\hline ERA-40 & 96 & 2 & $3.6 \pm 0.3$ & 4.9 & 0.079 & $0.9 \pm 0.3$ \\
\hline NCEP & 96 & 2 & $4.0 \pm 0.3$ & 4.3 & 0.065 & $1.1 \pm 0.3$ \\
\hline NCEP2 & 98 & 2 & $3.6 \pm 0.2$ & 4.6 & 0.070 & $0.9 \pm 0.3$ \\
\hline Model I & 109 & 3 & $3.4 \pm 0.3$ & 5.3 & 0.080 & $>0.3$ \\
\hline Model II & 64 & 3 & $3.4 \pm 0.3$ & 5.3 & 0.072 & $>0.2$ \\
\hline \multicolumn{7}{|l|}{ Zonal mean temperature } \\
\hline ERA-Interim & 84 & 2 & $3.4 \pm 0.2$ & 4.6 & 0.075 & $0.8 \pm 0.3$ \\
\hline ERA-40 & 95 & 2 & $3.7 \pm 0.3$ & 3.7 & 0.050 & $0.8 \pm 0.3$ \\
\hline NCEP & 92 & 2 & $3.7 \pm 0.2$ & 3.4 & 0.046 & $0.8 \pm 0.2$ \\
\hline NCEP (starting 1 January 1979) & 78 & 2 & $3.2 \pm 0.1$ & 3.2 & 0.045 & $0.7 \pm 0.3$ \\
\hline NCEP2 & 92 & 2 & $3.3 \pm 0.2$ & 3.7 & 0.055 & $0.9 \pm 0.1$ \\
\hline NCEP2 (single longitude) & 102 & 2 & $4.9 \pm 0.8$ & 3.9 & 0.079 & $>0.8$ \\
\hline Model I & 94 & 3 & $3.2 \pm 0.2$ & 5.0 & 0.072 & $0.8 \pm 0.3$ \\
\hline Model II & 38 & 3 & $3.0 \pm 0.6$ & 5.0 & 0.072 & $>0.2$ \\
\hline \multicolumn{7}{|l|}{ Zonal mean geopotential height } \\
\hline ERA-Interim & 91 & 3 & $3.6 \pm 0.2$ & 5.5 & 0.076 & $0.9 \pm 0.2$ \\
\hline ERA-40 & 88 & 2 & $3.3 \pm 0.2$ & 4.8 & 0.070 & $0.8 \pm 0.3$ \\
\hline NCEP & 101 & 2 & $3.4 \pm 0.3$ & 4.8 & 0.065 & $>0.3$ \\
\hline NCEP2 & 102 & 2 & $3.6 \pm 0.1$ & 4.6 & 0.076 & $>0.3$ \\
\hline Model I & 61 & 3 & $3.0 \pm 0.4$ & 5.1 & 0.073 & $0.6 \pm 0.2$ \\
\hline Model II & 41 & 3 & $3.0 \pm 0.5$ & 5.4 & 0.076 & $>0.1$ \\
\hline
\end{tabular}

\section{2) ATTRACTOR DIMENSIONS}

Figure 5a shows the trend of $C_{2}(m, \epsilon)$ for the ERAInterim zonal mean zonal wind. For small values of $\epsilon$, the slope of $C_{2}$ (inset) is dominated by noise, while for large values of $\epsilon$ the data points tend to fill the embedding space and the slopes of $C 2$, calculated for different $m$, do not converge. In the interval between $1<\epsilon<10$, the slopes show convergence to $D_{2}=3.5 \pm 0.2$. The convergence of the slopes as a function of the embedding dimension $m$ for the ERA-Interim zonal mean zonal wind to the value of 3.5 is shown in Fig. 5b. A summary of $D_{2}$ calculated for all the different variables in all the datasets is reported in Table 1, column 3 , where the errors are calculated as the standard deviation of the slopes in the interval chosen for the calculation of the correlation dimension, and is summarized in Fig. 5c (black dots). Results show that $D_{2}$ varies between 3 , obtained for the zonal mean temperature and zonal mean geopotential height for model II, as well as for the zonal mean geopotential height for model I, and 4, obtained for the NCEP zonal mean zonal wind. In particular, the results from the model integrations show that the correlation dimension does not seem to depend on the forcing specified and must thus be inherently linked to other forms of forcing (e.g., the interaction with topography). The calculation of the Lyapunov spectra shows that the lower bound $D_{2}>D_{\lambda}$ is satisfied for all variables and datasets (Table 1, column 2).

The Lyapunov spectrum allows us to calculate the Kaplan-Yorke dimension (12). Results (Table 1, column 4; see also Fig. 5c, open dots) show a range of values varying between 3.3 , corresponding to the NCEP zonal mean temperature, and 5.5, corresponding to the ERAInterim zonal mean geopotential height. The NCEP zonal mean temperature is characterized by a value of the maximum Lyapunov exponent that is much lower than the values assumed by the other variables and time series (Table 1, column 5).

The difference between the correlation and Lyapunov dimensions is generally smaller for the zonal mean temperature, for which case the dimensions calculated from the ERA-40 are consistent. The model runs generally show a larger difference between the two dimensions for all the variables and datasets.

\section{3) DYNAMICAL ENTROPIES}

For small values of $\epsilon$ and for values of $m$ smaller than the dimension of the attractor, for both deterministic and stochastic processes, the Renyi entropies vary as $h_{q}(m, \epsilon) \approx-\ln \epsilon+C$, where $C$ is a constant that assumes the name of conditional continuous entropy. However, for increasing values of $m$, for not too small values of $\epsilon$, 
(a)

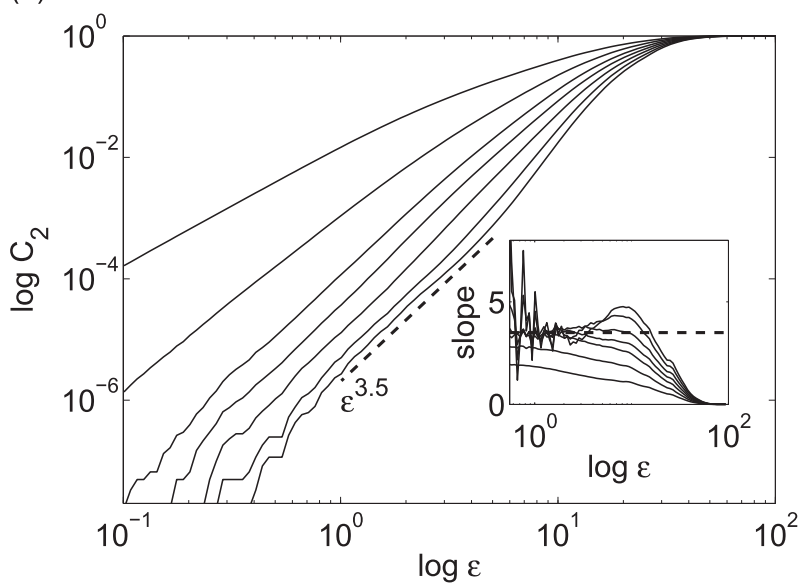

(b)

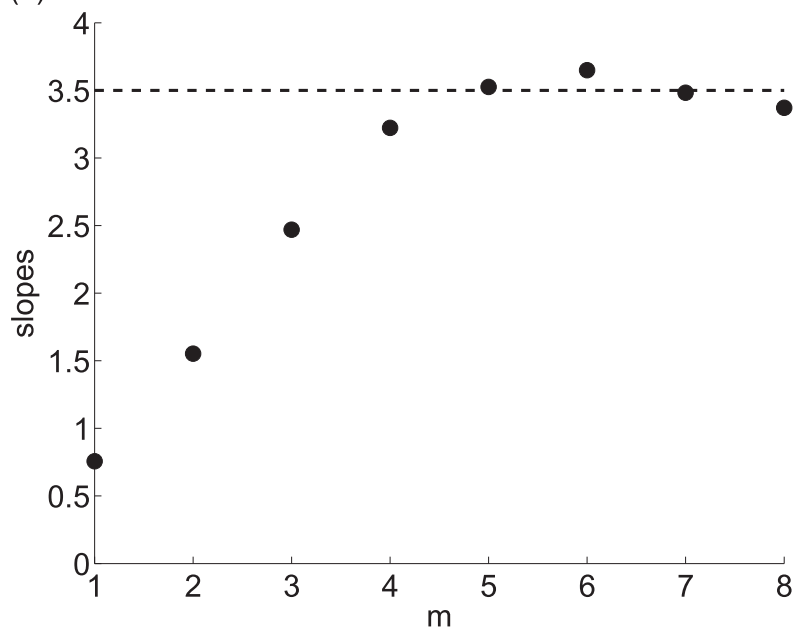

(c)

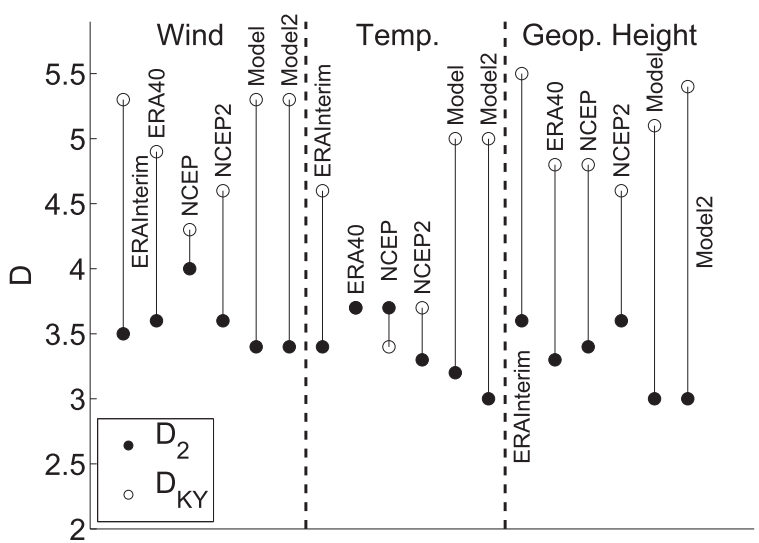

FIG. 5. (a) Correlation functions $C_{2}(m, \epsilon)$ and their slopes (inset) for zonal mean zonal wind at $60^{\circ} \mathrm{N}$ and $10 \mathrm{hPa}$ for ERA-Interim. (b) Slopes of the correlation functions $C_{2}$ as a function of the embedding dimension $m$ for zonal mean zonal wind at $60^{\circ} \mathrm{N}$ and $10 \mathrm{hPa}$ for ERA-Interim. The values converge to the value of 3.5 (dashed line). (c) Summary of the correlation dimension $\left(D_{2}\right)$ and the Kaplan-Yorke dimension $\left(D_{\mathrm{KY}}\right)$ found in the reanalyses and models for all three variables: (left to right) wind, temperature, and geopotential height. the values for $h_{q}$ converge to a finite value, thus allowing for the distinction between chaos and stochastic processes (Cencini et al. 2000). Figures 6a,b show the trend of $h_{2}(m, \epsilon)$ for the ERA-Interim zonal mean zonal wind and the NCEP zonal mean geopotential height, respectively. Results show a plateau in the curves that converge to a value of $0.9 \pm 0.3$ for the ERA-Interim zonal mean zonal wind, while the curves do not converge to a finite value for the NCEP zonal mean geopotential height. A summary of the correlation entropy for all the variables and datasets is reported in Table 1, column 6, and in Fig. 5 c. Results show that the entropy does not converge to a finite value for a number of datasets, making the correlation entropy a difficult indicator for the presence of chaotic behavior. For the datasets for which the entropy converges to a finite value, results range between 0.6 \pm 0.2 , corresponding to the time series of the zonal mean geopotential height for model I, and $1.1 \pm 0.3$, corresponding to the NCEP zonal mean zonal wind. The quantity $h_{2}$ seems to be generally higher for the zonal mean zonal wind and lower for the zonal mean geopotential height for all datasets.

\section{4) SENSITIVITY TESTS}

To assess the dependence of the results on the choice of the delay time $\tau$, calculations were repeated for the ERA-Interim zonal mean zonal wind choosing a delay time determined by the first zero crossing of the autocorrelation function of the time series. Despite the fact that the value of the delay time obtained from the autocorrelation function is less than half the value obtained from the first minimum of the mutual information, the results show good agreement for the calculation of the dimensions. The calculation of the correlation entropy, however, shows a lack of convergence and justifies the choice of using the first minimum of the mutual information, when it is present, for the time-delay parameter $\tau$.

To assess a possible impact of the positive trend in the variables present in the NCEP dataset, calculations were repeated for the subinterval from 1 January 1979 to 31 December 2012, consisting of 12415 data points. The results show a lower correlation dimension $D_{2}=3.4$ \pm 0.1 for the shorter time series, compared to the value of $D_{2}=3.7 \pm 0.2$ for the complete time series. The correlation entropies are in the same range, with value of $h_{2}=0.8 \pm 0.2$ for the shorter time series, while $h_{2}=$ $0.7 \pm 0.3$ for the complete time series.

Finally, the calculations were repeated using a single longitude point rather than a zonal mean for the temperature at $60^{\circ} \mathrm{N}$ and $10 \mathrm{hPa}$ for the NCEP2 time series. Results show that the relationship between the dimensions (13) does not hold. The analysis of the time series for the single point yields $D_{2}=4.9 \pm 0.8$ and 
(a)

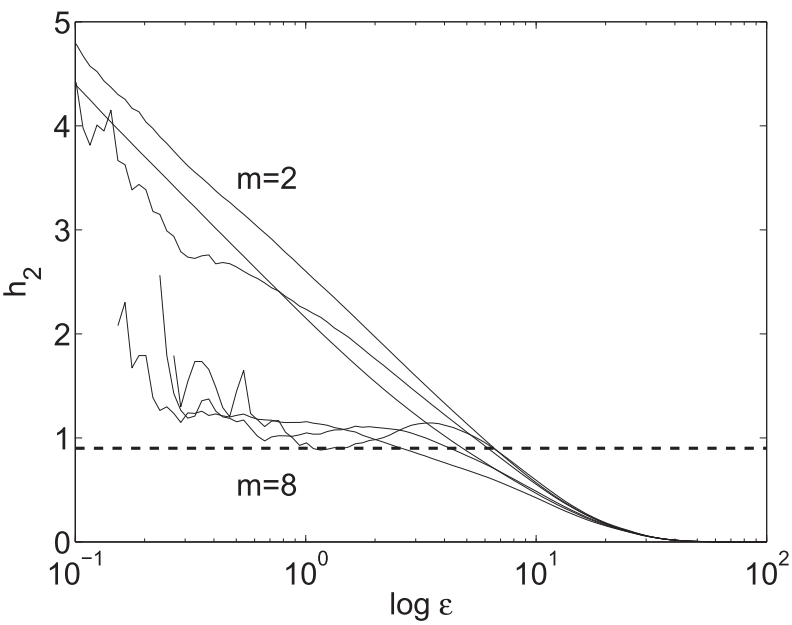

(b)

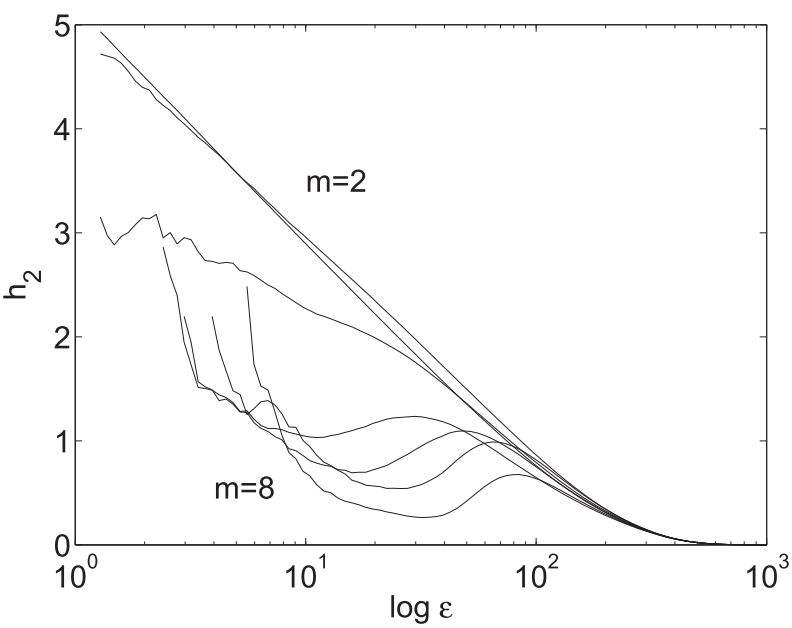

(c)

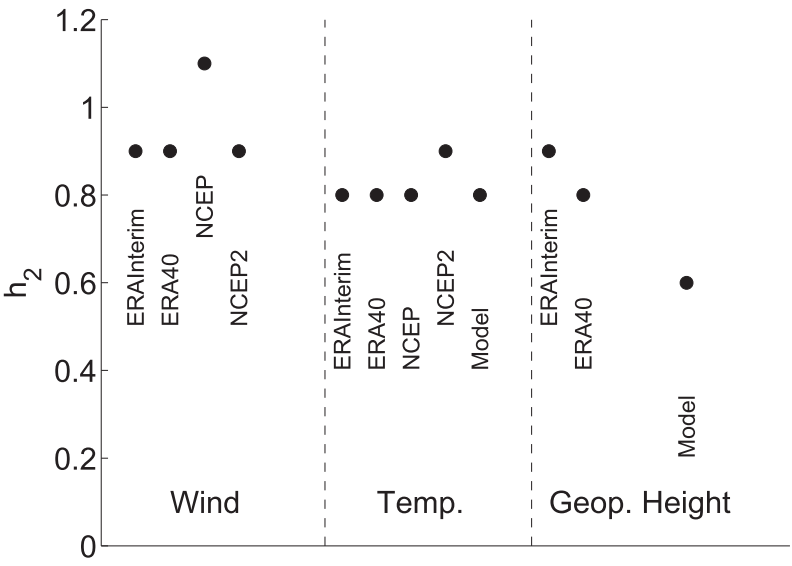

FIG. 6. (a) Correlation entropy $h_{2}(m, \epsilon)$ for zonal mean zonal wind at $60^{\circ} \mathrm{N}$ and $10 \mathrm{hPa}$ for ERA-Interim for $m=2-8$ (curves from top to bottom), and similarly (b) for zonal mean geopotential height at $60^{\circ} \mathrm{N}$ and $10 \mathrm{hPa}$ for NCEP. The quantity $h_{2}$ converges to $0.9 \pm 0.3$ (dashed line) for the ERA-Interim zonal mean zonal wind, while it does not converge for the NCEP zonal mean geopotential height. (c) Summary of the correlation entropy found in the reanalyses and models for all three variables: (left to right) wind, temperature, and geopotential height.
$D_{\mathrm{KY}}=3.9$, while the analysis of the zonal mean temperature yields $D_{2}=3.3 \pm 0.2$ and $D_{\mathrm{KY}}=3.7$. The analysis of the time series for the single longitude point is in addition characterized by a lack of convergence of the dynamical entropy. Studying a low-order primitive equation model of the atmospheric circulation (Lorenz 1980), Sundermeyer and Vallis (1993) found that the correlation dimension increases in presence of fast dynamics in the form of gravity waves. Further work is required to study the presence of signatures of fast dynamics that may not allow for the calculation of the dimension of the attractor of the system in the single longitude measurements.

\section{Discussion}

While the existence of a strange attractor for climate has proven difficult and is limited by a number of factors, the aims of this study are to search for a finite attractor for a specific climate subsystem, represented by extratropical Northern Hemisphere stratospheric variability, and to study the dependence of the dimension of the attractor on the choice of the analyzed variable, using four different reanalyses. Results are also tested for two model runs with different forcings in order to test the dependence of the dimension of the attractor to the seasonality of the forcing. The analysis of the time series yields a convergence to finite dimensions for all the different variables and datasets. Convergence of the dynamical entropies can however only be shown for a subset of variables and datasets. The different dimensions of the attractor follow the hierarchy of dimensions $D_{\lambda} \leq D_{2} \leq D_{1} \leq D_{0} \leq D_{\mathrm{KY}}$. Interestingly, however, results show a large difference in values between the correlation $\left(D_{2}\right)$ and the Lyapunov $\left(D_{\mathrm{KY}}\right)$ dimensions, suggesting the presence of a multifractal structure for the attractor associated with the variability of the system (Parisi and Frisch 1985; Halsey et al. 1986).

It is interesting to note how the time series generated from the idealized numerical model simulations forced by seasonally varying forcing and by permanent winter conditions compare with the results from the reanalyses. The model forced with a seasonal cycle exhibits a different frequency spectrum than the reanalyses, with slopes that are shallower than the reanalyses at low frequencies and steeper than the reanalyses at higher frequencies. Further, both model simulations show correlation dimensions that are lower (within the error bounds) than the values obtained from the reanalyses and Lyapunov dimensions higher than the values obtained from the reanalyses. The two model simulations thus exhibit a larger difference between the correlation and Lyapunov dimensions than the reanalyses, suggesting 
that the numerical integrations might possess a different multifractal structure than the observations. Interestingly, this result holds both for the seasonally forced and for the model forced with permanent winter conditions, suggesting that the characteristics of a possible attractor associated with the model variability must be influenced primarily by other types of forcing (e.g., by interactions with topography or by internal dynamics of the model). It would be interesting to compare the results reported here with the results of the same calculations conducted for the Southern Hemisphere, which exhibits a considerably different winter evolution in the stratosphere (Plumb 1989); that is, the stratospheric variability is not as strongly influenced by topographic forcing and SSW events are rare.

The results obtained in this study thus question the characteristics of a strange attractor for stratospheric variability, leaving open the question if the climate system can be modeled as a chaotic system or as a stochastic system (Hasselmann 1976) (i.e., from the knowledge of statistical, rather than deterministic, properties). On the other hand, elements such as the redness of the spectra and the deviation from the Gaussian tails for all the variables suggest that other processes, such as stable Levy processes (e.g., Penland and Ewald 2008), might be a possible representation of the system. Further work is required to determine the presence of multifractal structures and the correct statistical representation of the variability.

Acknowledgments. ERA-40 and ERA-Interim data have been obtained from the ECMWF Data Server. NCEP reanalysis data was obtained from the ESRL Data Server. DD would like to thank Alan Plumb for computer resources at MIT, where the model runs were performed; Greg Shomo for technical support; and Aditi Sheshadri for interpolating part of the model I data to pressure levels. The authors thank Johanna Baehr for helpful discussions of the manuscript and Edwin Gerber for helpful discussions on the trend in the reanalysis data. The authors thank three anonymous referees for comments that helped to improve the manuscript.

\section{REFERENCES}

Aurell, E., G. Boffetta, A. C. G. Paladin, and A. Vulpiani, 1997: Predictability in the large: An extension of the concept of Lyapunov exponent. J. Phys. Math. Gen., 30, 1-26.

Baldwin, M., and T. Dunkerton, 2001: Stratospheric harbingers of anomalous weather regimes. Science, 294, 581-584.

—, D. B. Stephenson, D. W. J. Thompson, T. J. Dunkerton, A. J. Charlton, and A. O'Neill, 2003: Stratospheric memory and skill of extended-range weather forecasts. Science, 301, 636-640.
Birner, T., and P. Williams, 2008: Sudden stratospheric warmings as noise-induced transitions. J. Atmos. Sci., 65, 3337-3343.

Black, R., B. McDaniel, and W. A. Robinson, 2006: Stratospheretroposphere coupling during spring onset. J. Climate, 19, 4891-4901.

Boffetta, G., A. Crisanti, F. Paparella, A. Provenzale, and A. Vulpiani, 1998: Slow and fast dynamics in coupled systems: A time series analysis view. Physica D, 116, 301-312.

Carbonell, M., R. Oliver, and J. L. Ballester, 1994: A search for chaotic behaviour in solar activity. Astron. Astrophys., 290, 983-994.

Cencini, M., M. Falcioni, H. Kantz, E. Olbrich, and A. Vulpiani, 2000: Chaos or noise-Difficulties of a distinction. Phys. Rev., 62E, 427-437.

Charney, J., and P. Drazin, 1961: Propagation of planetaryscale disturbances from the lower into the upper atmosphere. J. Geophys. Res., 66, 83-109.

Christiansen, B., 2000: Chaos, quasiperiodicity, and interannual variability: Studies of a stratospheric vacillation model. J. Atmos. Sci., 57, 3161-3173.

- 2009: Is the atmosphere interesting? A projection pursuit study of the circulation in the Northern Hemisphere winter. J. Climate, 22, 1239-1254.

Dee, D. P., and Coauthors, 2011: The ERA-Interim reanalysis: Configuration and performance of the data assimilation system. Quart. J. Roy. Meteor. Soc., 137, 553-597.

Eckmann, J. P., and D. Ruelle, 1985: Ergodic theory of chaos and strange attractors. Rev. Mod. Phys., 57, 617-655.

Elsner, J. B., and A. A. Tsonis, 1992: Nonlinear prediction, chaos, and noise. Bull. Amer. Meteor. Soc., 73, 49-60.

Esler, J. G., and N. J. Matthewman, 2011: Stratospheric sudden warmings as self-tuning resonances. Part II: Vortex displacement events. J. Atmos. Sci., 68, 2505-2523.

Essex, C., and M. A. H. Nerenberg, 1991: Comments on 'Deterministic chaos: The science and the fiction' by D. Ruelle. Proc. Roy. Soc. London, 453A, 287-292.

Fraedrich, K., 1986: Estimating the dimensions of weather and climate attractors. J. Atmos. Sci., 43, 419-432.

_ 1987: Estimating weather and climate predictability on attractors. J. Atmos. Sci., 44, 722-728.

Fraser, A. M., and H. L. Swinney, 1986: Independent coordinates for strange attractors from mutual information. Phys. Rev., 33A, 1134-1140.

Gerber, E. P., and L. M. Polvani, 2009: Stratosphere-troposphere coupling in a relatively simple AGCM: The importance of stratospheric variability. J. Climate, 22, 1920-1933.

,-- , and D. Ancukiewicz, 2008: Annular mode time scales in the Intergovernmental Panel on Climate Change Fourth Assessment Report models. Geophys. Res. Lett., 35, L22707, doi:10.1029/2008GL035712.

Grassberger, P., 1986: Do climatic attractors exist? Nature, 323, 609-612.

— tors. Phys. Rev. Lett., 50, 346-349.

- and $-1983 \mathrm{~b}$ : Estimation of the Kolmogorov entropy from a chaotic signal. Phys. Rev., 28A, 2591-2593.

$\longrightarrow$, and — 1983c: Measuring the strangeness of strange attractors. Physica D, 9, 189-208.

Halsey, T., M. Jensen, L. Kadanoff, I. Procaccia, and B. Shraimann, 1986: Fractal measures and their singularities: The characterization of strange sets. Phys. Rev., 33A, 1141-1151.

Hardiman, S. C., and P. H. Haynes, 2008: Dynamical sensitivity of the stratospheric circulation and downward influence of upper level perturbations. J. Geophys. Res., 113, D23103, doi:10.1029/ 2008JD010168. 
Hasselmann, K., 1976: Stochastic climate models. 1. Theory. Tellus, 28, 473-485.

Haynes, P. H., 2005: Stratospheric dynamics. Annu. Rev. Fluid Mech., 37, 263-293.

Held, I. M., and M. J. Suarez, 1994: A proposal for the intercomparison of the dynamical cores of atmospheric general circulation models. Bull. Amer. Meteor. Soc., 75, 1825-1830.

Hentschel, H. G. E., and I. Procaccia, 1983: The infinite number of generalized dimensions of fractals and strange attractors. Physica D, 8, 435-444.

Hirota, I., T. Hirooka, and M. Shiotani, 1983: Upper stratospheric circulations in the two hemispheres observed by satellites. Quart. J. Roy. Meteor. Soc., 109, 443-454.

Holton, J., and C. Mass, 1976: Stratospheric vacillation cycles. J. Atmos. Sci., 33, 2218-2225.

Jucker, M., S. Fueglistaler, and G. Vallis, 2013:Maintenance of the stratospheric structure in an idealized general circulation model. J. Atmos. Sci., 70, 3341-3358.

Kalnay, E., and Coauthors, 1996: The NCEP/NCAR 40-Year Reanalysis Project. Bull. Amer. Meteor. Soc., 77, 437-471.

Kanamitsu, M., W. Ebisuzaki, J. Woollen, S.-K. Yang, J. J. Hnilo, M. Fiorino, and G. L. Potter, 2002: NCEP-DOE AMIP-II Reanalysis (R-2). Bull. Amer. Meteor. Soc., 83, 1631-1643.

Kaplan, J. L., and J. A. Yorke, 1979: Chaotic behavior of multidimensional difference equations. Functional Differential Equations and Approximations of Fixed Points, H.-O. Peitgen and H.-O. Walther, Eds., Lecture Notes in Mathematics, Vol. 730, Springer, 204-227.

Keppenne, C. L., and C. Nicolis, 1989: Global properties and local structure of the weather attractor over Western Europe. J. Atmos. Sci., 46, 2356-2370.

Kistler, R., and Coauthors, 2001: The NCEP-NCAR 50-Year Reanalysis: Monthly means CD-ROM and documentation. Bull. Amer. Meteor. Soc., 82, 247-268.

Kolmogorov, A. N., 1958: A new invariant of transitive dynamical systems. Dokl. Akad. Nauk SSSR, 119, 861-864.

Kushner, P., and L. M. Polvani, 2006: Stratosphere-troposphere coupling in a relatively simple AGCM: Impact of the seasonal cycle. J. Climate, 19, 5721-5727.

Labitzke, K., 1981: Stratospheric-mesospheric midwinter disturbances: A summary of observed characteristics. J. Geophys. Res., 86 (C10), 9665-9678.

Lorenz, E. N., 1960: Maximum simplification of the dynamic equations. Tellus, 12, 243-254.

_ 1980: Attractor sets and quasigeostrophic equilibrium. J. Atmos. Sci., 37, 1685-1699.

_ 1991: Dimension of weather and climate attractors. Nature, 353, 241-244.

Mandelbrot, B. B., 1977: Fractals: Form, Chance and Dimension. W.H. Freeman and Company, 365 pp.

_ , and J. W. VanNess, 1968: Fractional Brownian motions, fractional noises and applications. SIAM Rev., 10, 422-437.

McInturff, R., 1978: Stratospheric warming: Synoptic, dynamic and general circulation aspects. NASA Reference Publ. 1017, 175 pp

Nicolis, C., and G. Nicolis, 1984: Is there a climatic attractor? Nature, 311, 529-532.

Osborne, A. R., and A. Provenzale, 1989: Finite correlation dimensions for stochastic systems with power-law spectra. Physica D, 35, 357-381.

Parisi, G., and U. Frisch, 1985: Fully developed turbulence and intermittency. Turbulence and Predictability in Geophysical Fluid Dynamics and Climate Dynamics, M. Ghil, Ed., Elsevier Science, $84-88$
Penland, C., and B. D. Ewald, 2008: On modelling physical systems with stochastic models: Diffusion versus Levy processes. Philos. Trans. Roy. Soc. London, A366, 2457-2476.

Plumb, R. A., 1989: On the seasonal cycle of stratospheric planetary waves. Pure Appl. Geophys., 130, 233-242.

Polvani, L. M., and P. J. Kushner, 2002: Tropospheric response to stratospheric perturbations in a relatively simple general circulation model. Geophys. Res. Lett., 29, 1114, doi:10.1029/ 2001GL014284

Renyi, A., 1971: Probability Theory. Dover Publications, 672 pp.

Ruelle, R., 1990: Deterministic chaos: The science and the fiction. Proc. Roy. Soc. London, 427A, 241-248.

Ruzmaikin, A., J. Lawrence, and C. Cadavid, 2003: A simple model of stratospheric dynamics including solar variability. J. Climate, 16, 1593-1600.

Sano, M., and Y. Sawada, 1985: Measurement of the Lyapunov spectrum from a chaotic time series. Phys. Rev. Lett., 55, 1082-1085.

Scherhag, R., 1952: Die explosionsartige Stratosphärenerwärmung des Spätwinters 1951/52. Ber. Dtsch. Wetterdienstes, 38, 51-63. , 1953: Rätselhafte Temperatursprünge in der Stratosphäre. Umschau, 53, 646-648.

Scott, R. K., and L. M. Polvani, 2006: Internal variability of the winter stratosphere. Part I: Time-independent forcing. $J$. Atmos. Sci., 63, 2758-2776.

, - - and D. W. Waugh, 2008: Internal variability of the winter stratosphere. Part II: Time-dependent forcing. $J$. Atmos. Sci., 65, 2375-2388.

Sheshadri, A., R. A. Plumb, and D. I. V. Domeisen, 2014: Can the delay in Antarctic polar vortex breakup explain recent trends in surface westerlies? J. Atmos. Sci., 71, 566-573, doi:10.1175/ JAS-D-12-0343.1.

Sinai, Y. G., 1959: On the notion of entropy of a dynamical system. Dokl. Akad. Nauk SSSR, 124, 768-771.

Sundermeyer, M., and G. K. Vallis, 1993: Correlation dimensions of primitive equation and balanced models. J. Atmos. Sci., 50, 2556-2564.

Takens, F., 1981: Detecting strange attractors in turbulence. $D y$ namical Systems and Turbulence, D. A. Rand and L.-S. Young, Eds., Springer-Verlag, 366-381.

Theiler, J., 1991: Some comments on the correlation dimension of $1 / f^{\alpha}$ noise. Phys. Lett., 155A, 488-492.

Tsonis, A. A., J. B. Elsner, and K. P. Georgakakos, 1993: Estimating the dimension of weather and climate attractors: Important issues about the procedure and interpretation. J. Atmos. Sci., 50, 2549-2555.

Uppala, S. M., and Coauthors, 2005: The ERA-40 Re-Analysis. Quart. J. Roy. Meteor. Soc., 131, 2961-3012.

Vautard, R., and M. Ghil, 1989: Singular spectrum analysis in nonlinear dynamics, with applications to paleoclimatic time series. Physica D, 35, 395-424.

Weber, R. O., P. Talkner, G. Stefanicki, and L. Arvisais, 1995: Search for finite dimensional attractors in atmospheric turbulence. Bound.-Layer Meteor., 73, 1-14.

Whitney, H., 1936: Differentiable manifolds. Ann. Math., 37, 645-680.

Yoden, S., 1987a: Bifurcation properties of a stratospheric vacillation. J. Atmos. Sci., 44, 1723-1733.

1987b: Dynamical aspects of stratospheric vacillations in a highly truncated model. J. Atmos. Sci., 44, 3683-3695.

, 1990: An illustrative model of seasonal and interannual variations of the stratospheric circulation. J. Atmos. Sci., 47, 1845-1853.

Zeng, X., R. A. Pielke, and R. Eykholt, 1992: Estimating the fractal dimension and predictability of the atmosphere. J. Atmos. Sci., 49, 649-659. 